\title{
SUMS OF SETS OF CONTINUED FRACTIONS
}

\author{
T. W. CUSICK AND R. A. LEE
}

ABSTRACT. For each integer $k \geqq 2$, let $S(k)$ denote the set of real numbers $\alpha$ such that $0 \leqq \alpha \leqq k^{-1}$ and $\alpha$ has a continued fraction containing no partial quotient less than $k$. It is proved that every number in the interval $[0,1]$ is representable as a sum of $k$ elements of $S(k)$.

For each integer $k \geqq 2$, let $S(k)$ denote the set of real numbers $\alpha$ such that $0 \leqq \alpha \leqq k^{-1}$ and $\alpha$ has a continued fraction containing no partial quotient less than $k$ (here 0 is to be regarded as the reciprocal of an infinite partial quotient, so 0 belongs to $S(k)$ ). Define the sum $A+B$ of two point sets $A$ and $B$ to be the set of all $a+b$, where $a$ is in $A$ and $b$ is in $B$. Define the sum $A+A+\cdots+A$ ( $n$ summands) inductively for each integer $n \geqq 2$, and let $n A$ denote the resulting point set.

Theorem 1 of paper [1] by the first author is equivalent to the assertion that $S(2)+S(2)=[0,1]$. In this paper we prove the following generalization of this result:

THEOREM. For each integer $k \geqq 2, k S(k)=[0,1]$.

We make use of the fact that for each integer $k \geqq 2$ the set $S(k)$ may be obtained from $\left[0, k^{-1}\right]$ by the removal of a suitable infinite set of disjoint open intervals. In fact, as explained in [1], $S(k)$ belongs to the class of Cantor point sets, which are defined by the following procedure: Take a closed interval $A=[x, x+a]$ on the real line, and remove from it a middle open interval $A_{12}=\left(x+a_{1}, x+a_{1}+a_{12}\right)$; two closed intervals $A_{1}=\left[x, x+a_{1}\right]$ and $A_{2}=\left[x+a_{1}+a_{12}, x+a\right]$ remain. The second stage of the procedure is the removal of middle open intervals from $A_{1}$ and $A_{2}$. The process is continued, so the $n$th stage of the procedure is the removal of $2^{n-1}$ middle open intervals. The set which results in the limit, when every closed interval which arises has been subdivided, is a Cantor point set.

To begin the procedure for obtaining $S(k)$ as a Cantor point set, we take $A=\left[0, k^{-1}\right]$. Let $\mathrm{CF}\left(0, a_{1}, a_{2}, \cdots\right)$ denote the continued fraction with partial quotients $0, a_{1}, a_{2}, \ldots$. In defining the sub-

Presented to the Society, April 9, 1971; received by the editors November 2, 1970.

A MS 1970 subject classifications. Primary 10F20, 10J99; Secondary 10K15.

Key words and phrases. Continued fractions, Cantor sets, sums of sets. 
divisions of $A$ which produce $S(k)$, two types of intervals with rational endpoints need to be considered. Intervals of the first type have the form

$$
\left[\mathrm{CF}\left(0, a_{1}, \cdots, a_{n}\right), \mathrm{CF}\left(0, a_{1}, \cdots, a_{n+1}\right)\right]
$$

where $n$ is an even integer and $a_{i} \geqq k(i=1,2, \cdots)$; intervals of the second type have the form

$$
\left[\mathrm{CF}\left(0, a_{1}, \cdots, a_{n}\right), \mathrm{CF}\left(0, a_{1}, \cdots, a_{n-1}\right)\right]
$$

where $n$ is an even integer and $a_{i} \geqq k(i=1,2, \cdots)$. Thus $A$ is an interval of the first type with $n=0$.

In the subdivision process, the interval

$$
\left(\mathrm{CF}\left(0, a_{1}, \cdots, a_{n}, a_{n+1}+1\right), \mathrm{CF}\left(0, a_{1}, \cdots, a_{n+1}, k\right)\right)
$$

is removed from each interval (1) of first type and the interval

$$
\left(\mathrm{CF}\left(0, a_{1}, \cdots, a_{n}, k\right), \mathrm{CF}\left(0, a_{1}, \cdots, a_{n-1}, a_{n}+1\right)\right)
$$

is removed from each interval (2) of second type. In both cases the removal of the middle interval leaves behind an interval of first type on the left side and an interval of second type on the right side. Therefore the subdivision process can be continued, and the resulting Cantor point set is $S(k)$. We shall call this procedure the Cantor dissection process for obtaining $S(k)$.

Marshall Hall, Jr. gave a sufficient condition for the sum of two Cantor point sets in the intervals $B_{1}$ and $B_{2}$ to cover the whole interval $B_{1}+B_{2}[2$, p. 968]. This condition was used in the proof of Theorem 1 of [1]. The proof of the theorem of this paper uses a general sufficient condition for the sum of $k$ copies of a point set contained in an interval $A$ to cover the whole interval $k A$.

Given any interval $I$ of real numbers, let $|I|$ denote the length of the interval. Let $q_{j}$ denote the denominator of $\operatorname{CF}\left(0, a_{1}, \cdots, a_{j}\right)$ in lowest terms. We require four preliminary lemmas.

LEMMA 1. Let I be any one of the open intervals removed in the Cantor dissection process for obtaining $S(k)$. Let $M$ denote the closed interval from which $I$ is removed in the dissection process, and let $M_{1}$ and $M_{2}$ denote the left-hand and right-hand intervals, respectively, into which $M$ is divided by the removal of $I$. Then

$$
(k-1)\left|M_{j}\right| \geqq|I| \quad(j=1,2) .
$$

Proof. A simple calculation shows that the length of the interval (1) of first type is $\left(q_{n} q_{n+1}\right)^{-1}$ and that the ratios of the length 
of the excluded interval (3) to the lengths of the retained intervals on the left and right, respectively, are $(k-1) q_{n} /\left(k q_{n+1}+q_{n}\right)$ and $(k-1) q_{n+1} /\left(q_{n+1}+q_{n}\right)$. These ratios are always less than 1 and $k-1$, respectively. Similarly, the length of the interval (2) of second type is $\left(q_{n-1} q_{n}\right)^{-1}$ and the ratios of the length of the excluded interval (4) to the lengths of the retained intervals on the left and right, respectively, are $(k-1) q_{n} /\left(q_{n}+q_{n-1}\right)$ and $(k-1) q_{n-1} /\left(k q_{n}+q_{n-1}\right)$. These ratios are always less than $k-1$ and 1 , respectively. This proves (5).

Lemma 2. Let $A_{1}, A_{2}, \cdots, A_{n}$ be any bounded closed intervals of real numbers. Suppose that an open interval $I$ is removed from the middle of $A_{1}$, leaving two closed intervals $A_{11}$ and $A_{12}$ on the left and right, respectively. If $\sum_{i=2}^{n}\left|A_{i}\right| \geqq|I|$, then

$$
A_{1}+A_{2}+\cdots+A_{n}=\left(A_{11} \cup A_{12}\right)+A_{2}+\cdots+A_{n} .
$$

Proof. The sum $A_{11}+\sum_{i=2}^{n} A_{i}$ is a closed interval with the same left-hand endpoint, say $L$, as $\sum_{i=1}^{n} A_{i}$ and with right-hand endpoint $L+\left|A_{11}\right|+\sum_{i=2}^{n}\left|A_{i}\right|$. The sum $A_{12}+\sum_{i=2}^{n} A_{i}$ is a closed interval with the same right-hand endpoint as $\sum_{i=1}^{n} A_{i}$ and with left-hand endpoint $L+\left|A_{11}\right|+|I|$. Thus these two intervals cover $\sum_{i=1}^{n} A_{i}$ if and only if they overlap, that is, if and only if

$$
L+\left|A_{11}\right|+|I| \leqq L+\left|A_{11}\right|+\sum_{i=2}^{n}\left|A_{i}\right| .
$$

This proves the lemma.

Lemma 3. Let $B$ be the union of a finite number of disjoint bounded closed intervals $A_{1}, A_{2}, \cdots, A_{r}$ of real numbers. Suppose that an open interval $I$ is removed from the middle of $A_{1}$, leaving two closed intervals $A_{r+1}$ and $A_{r+2}$ on the left and right, respectively. Let $B^{*}=\bigcup_{i=2}^{r+2} A_{i}$. If

$$
(n-1)\left|A_{i}\right| \geqq|I| \quad(2 \leqq i \leqq r+2),
$$

then $n B^{*}=n B$.

Proof. It follows from (6) and Lemma 2 that for any choice of integers $f(i)(2 \leqq i \leqq n)$ satisf ying $2 \leqq f(i) \leqq r+2$, we have

$$
A_{1}+\sum_{i=2}^{n} A_{f(i)}=\left(A_{r+1} \cup A_{r+2}\right)+\sum_{i=2}^{n} A_{f(i)} .
$$

Define $A_{1}^{*}=A_{r+1} \cup A_{r+2}$ and $A_{i}^{*}=A_{i}(2 \leqq i \leqq r)$. For any choice of integers $g(i)(1 \leqq i \leqq n)$ satisfying $1 \leqq g(i) \leqq r$ and $g(i)=1$ for at least one $i$, the set $\sum_{i=1}^{n} A_{g(i)}^{*}$ can be written as a union of sets of the form $A_{1}^{*}+\sum_{i=2}^{n} A_{f(i)}$, where $2 \leqq f(i) \leqq r+2$ for each $i, 2 \leqq i \leqq n$. Thus (7) 
implies that for any choice of integers $g(i)(1 \leqq i \leqq n)$ satisfying $1 \leqq g(i) \leqq r$, we have

$$
\sum_{i=1}^{n} A_{o(i)}^{*}=\sum_{i=1}^{n} A_{o(i)}
$$

This implies $n B^{*}=n B$, as required.

Lemma 4. If $C_{1}, C_{2}, \cdots$ is a sequence of bounded closed sets of real numbers and $C_{i}$ contains $C_{i+1}$ for $i=1,2, \cdots$, then

$$
n \bigcap_{i=1}^{\infty} C_{i}=\bigcap_{i=1}^{\infty} n C_{i} \text {. }
$$

Proof. For any $i$, the set $n C_{i}$ contains the left side of (8), so the right side of (8) clearly contains the left. In order to prove the opposite containment, let $t$ be any number in $\bigcap_{i=1}^{\infty} n C_{i}$.

Define for each $i=1,2, \cdots$,

$$
V_{i}=\left\{\left(x_{1}, x_{2}, \cdots, x_{n}\right): x_{j} \text { is in } C_{i} \text { for } 1 \leqq j \leqq n\right\}
$$

and define

$$
T=\left\{\left(x_{1}, x_{2}, \cdots, x_{n}\right): \sum_{i=1}^{n} x_{i}=t\right\} .
$$

Since $V_{i}$ is a bounded closed set and $T$ is closed, the set $T \cap V_{i}$ is bounded, closed and nonempty. Also $T \cap V_{i}$ contains $T \cap V_{i+1}$ for $i=1,2, \cdots$, so by the Cantor intersection theorem

$$
\bigcap_{i=1}^{\infty}\left(T \cap V_{i}\right)=T \cap \bigcap_{i=1}^{\infty} V_{i}
$$

is a nonempty set. If $\left(t_{1}, t_{2}, \cdots, t_{n}\right)$ is any member of this set, then each $t_{i}$ is in $\bigcap_{i=1}^{\infty} C_{i}$ and $\sum_{i=1}^{n} t_{i}=t$. Hence $t$ belongs to $n \bigcap_{i=1}^{\infty} C_{i}$, and this proves the lemma.

Proof of ThEOREM. We take $S_{0}=\left[0, k^{-1}\right]$ and define a sequence of sets $S_{0}, S_{1}, S_{2}, \ldots$ with the following properties:

$$
\begin{gathered}
S_{i} \text { contains } S_{i+1} \quad(i=0,1,2, \cdots) ; \\
\bigcap_{i=0}^{\infty} S_{i}=S(k) ;
\end{gathered}
$$

and

$$
k S_{i}=k S_{i+1} \quad(i=0,1,2, \cdots)
$$


We saw earlier that $S(k)$ may be obtained from $S_{0}$ by removing an infinite set of disjoint open intervals, namely the set of all open intervals of form (3) or (4) with each $a_{i} \geqq k$. We can arrange this set of open intervals in order of decreasing length (if several intervals have the same length, we can take them in any order, say from left to right in $S_{0}$ ). Let the resulting sequence of intervals be $D_{0}, D_{1}, D_{2}, \cdots$ and define $S_{i}$ to be $S_{i-1}$ with $D_{i-1}$ removed $(i=1,2, \cdots)$. Clearly the sequence $S_{0}, S_{1}, S_{2}, \cdots$ has the properties (9) and (10) above.

In order to show that (11) holds, we apply Lemma 3 with $n=k$ and, successively, $B=S_{i}, B^{*}=S_{i+1}, I=D_{i}(i=0,1,2, \cdots)$. The following considerations show that the condition (6) holds for each application of the lemma.

For each $i=0,1,2, \cdots, S_{i+1}$ is obtained from $S_{i}$ by removing $D_{i}$ from some closed interval, say $H_{i}$, contained in $S_{i}$. The removal of $D_{i}$ splits $H_{i}$ into two closed intervals, say $H_{i 1}$ and $H_{i 2}$, to the left and right, respectively, of $D_{i}$. If (6) holds for $B=S_{i-1}, B^{*}=S_{i}, I=D_{i-1}$, then, since $\left|D_{i-1}\right| \geqq\left|D_{i}\right|$, in order to prove (6) for $B=S_{i}, B^{*}=S_{i+1}$, $I=D_{i}$, it suffices to verify

$$
(k-1)\left|H_{i j}\right| \geqq\left|D_{i}\right| \quad(j=1,2) .
$$

We take $I=D_{i}$ in (5), so that $M_{1}$ and $M_{2}$ are closed intervals adjacent to $D_{i}$ on the left and right, respectively. If $H_{i j}$ contains or is equal to $M_{j}$, then (12) follows from (5).

If $H_{i j}$ is strictly contained in $M_{j}$, then there is some interval $D_{m}$ with $m<i$ adjacent to $H_{i j}$ on the side opposite $D_{i}$, and $D_{m}$ intersects $M_{j}$. Therefore $D_{m}$ must be removed after $D_{i}$ in the Cantor dissection process for obtaining $S(k)$. The removal of $D_{m}$ in the Cantor dissection process leaves behind two closed intervals, one adjacent to each end of $D_{m}$. Let $N$ denote the interval of these two which is between $D_{m}$ and $D_{i}$. Since $H_{i j}$ must contain $N$, we have

$$
(k-1)\left|H_{i j}\right| \geqq(k-1)|N| \geqq\left|D_{m}\right| \geqq\left|D_{i}\right| ;
$$

the second inequality follows from (5) with $I=D_{m}$ and the third inequality follows from the fact that $m<i$. This completes the proof of (12). It is not difficult to show that in fact the set $M$ of Lemma 1 always contains $H_{i}$, so the situation discussed in this paragraph never occurs. However, we do not need this, and the proof given has the advantage of avoiding any detailed appeal to properties of the Cantor dissection process.

Using (12), we prove by induction that (6) holds in Lemma 3 with $B=S_{i}, B^{*}=S_{i+1}, I=D_{i}(i=0,1,2, \cdots)$. This completes the proof of (11). 
Since $k S_{0}=[0,1],(9)$ and (11) imply $[0,1]=\bigcap_{i=0}^{\infty} k S_{i}$. Now (9), (10) and Lemma 4 give the theorem.

The theorem is best possible in the sense that for any $k \geqq 2$ and any $j<k, j S(k)=[0,1]$ is false; for we have trivially that $j S(k)$ is contained in $j S_{0}=[0, j / k]$.

\section{REFERENCES}

1. T. W. Cusick, Sums and products of continued fractions, Proc. Amer. Math. Soc. 27 (1971), 35-38.

2. Marshall Hall, Jr., On the sum and product of continued fractions, Ann. of Math. (2) 48 (1947), 966-993. MR 9, 226.

State University of New York at Buffalo, Buffalo, New York 14226 